\title{
Correlation of internet addiction with anxiety, depression and personality traits -Upper Egypt study
}

\author{
Hemaid Mustafa Azab *, Safeya Mahmoud Ahmed Effat **, Hanan Yousef \\ Aly***, Osama Abd Alreheem Mahmoud****. \\ * Professor of Neuropsychiatry- faculty of medicine-Sohag University \\ ** Professor of Psychiatry- faculty of medicine-Ain Shams University \\ *** Lecturer of Neuropsychiatry- faculty of Medicine-Sohag University \\ ****Assistant Lecturer of Neuropsychiatry -faculty of Medicine-Sohag University
}

\begin{abstract}
Objective:The aim of the study was to investigate the relationship of Anxiety, Depression and personality traitswith Internet addiction (IA) inuniversity students. Methods:A cross-sectional survey was conducted on a random sample of 588 students selected from four faculties in Sohag University. IAwas assessed by the 20item Young Internet Addiction Test (YIAT). Information was also collected on socio-demographics, university-related factors and Internet usage patterns. Eysenck personality questionnaire (EPQ)used for Assessment of the personality traits. Taylor's Manifest Anxiety Scale was used as a general indicator of anxiety.Beck depression inventorywas used for measuring the existence and the severity of depression.
\end{abstract}

Results:Findings showed that the prevalence of problematic internet use (PIU)among the studied population was $35.2 \%$.About $86.9 \%$ of Students with PIU significantly had anxietyalso, $85.02 \%$ of Students with PIU significantly had Depression . PIU significantly had high score in Psychoticism and Neuroticism and low score in Lie and Extraversion.

Conclusion:PIU prevalent disorder among undergraduate university studentsinSohag. Clinicians and health professionals should be aware of the risk of PIUin students with Anxiety ,Depressionand abnormal personality triats.

Keywords: Internet Addiction, prevalence, Depression,Anxietyand personality

\section{INTRODUCTION}

There is no doubt that the internet has many uses including access to knowledge, research, communication, entertainment, shopping ,social platforms, and so on. ${ }^{[1]}$ However, it still may cause physical and psychological harms. ${ }^{[2]}$ Internet addiction can be defined as a maladaptive pattern of internet use leading to clinically significant impairment or distress . [3] The prevalence of internet addiction range from $0.3 \%$ to $38 \%$ in many studies , and this discrepancy might be due methodological variation in diagnosis and diversity in use of diagnostic instruments ${ }^{[4]}$ Previous studies about Internet addiction have investigated several associated psychological variables such as anxiety, depression, and interpersonal relations ${ }^{[5]}$ Many studies on Internet addiction focus on internal and individual factors that may predispose individuals to problematic Internet use ${ }^{[6]}$ Individuals with higher negative emotions and inward thinking are more susceptible to overuse of the Internet $\quad{ }^{[7]}$ Most of problems associated with Internet addiction, such as antisocial tendencies, social anxiety, and aggression, may returned back to maladaptive personality disorders described in the psychoticism, extraversion, and neuroticism $(\mathrm{P}-\mathrm{E}-\mathrm{N})$ model. ${ }^{[8]}$ 
SOHAG MEDICAL JOURNAL

Vol. 23 No.1 Jan 2019

Rationale of work:

Internet addiction among university students may be associated with many psychiatric problems including depression and social anxiety and personality disorder.These psychiatric problems are not well revealed, at least in the Egyptian university students .So, we want to research for these specific psychiatric problems in university students who use the internet .

\section{Aim of the work:}

To explore the relationship between internet addiction and anxiety, depression, personality traits among sample of Egyptian university students.

\section{METHOD:}

Study design:A cross-sectional survey was conducted from February 2017 to January 2018.

Site of study:Four Faculties inSohag University (Faculty of Veterinary Medicine, Faculty of Science, Faculty of Commerce and Faculty of Arts) were chosen to be involved in the current study.

\section{Study participants and sampling:}

The sample size was calculated to be 600 students as calculated using Open EPI program. By Using a multistage clustering sampling method, we selected the students for our study as follows: The first stage:Two practical faculities (faculty of Veterinary Medicine and faculty of Science) and Two theoretical faculities (faculty of Arts and faculty of Commerce) were choosen from Sohage university following simple random sampling technique.The second stage: The sample size divided on both practical and theoretical faculities with 400 students from theoretical faculities and 200 students from practical faculities according to stratified cluster random sampling technique. 12 students were excluded on the
Correlation of internet addiction

Osama Abd Alreheem Mahmoud.et al

basis of incomplete questionnaires and the study included 588 students.

\section{Study instrument}

All students were subjected to the following procedures:

1-Self- administered questionnaire for Assesment of; Socio-demographic ,academic data of the student and Patterns of internet use.

2-Assesment of the degree of internet addiction: By using Young internet addiction test ${ }^{[1]} \mathrm{We}$ used the Arabic version translated byHawi. ${ }^{[9]}$ The IAT includes 20 items, each of which is rated on a six-point Likert scaleIt has ascore range from 0 to 100 .In the present study The following cut-off points were applied to the total YIAT score:Normal Internet use or non problematic Internet use : scores 0-49 and Potential problematic Internet use (PIUs): scores equal to or over 50.

3-Taylor's Manifest Anxiety Scale (TMAS) ${ }^{[10]}$ : As a general indicator of anxiety as a personality trait. We used the Arabic version translated byFahmi et al. ${ }^{[11]}$ It consisted of 50 true or false questions.It has Score range from 0 to 50: no anxiety (0 -16), mild anxiety (17 - 24), moderate anxiety (25 -35) and sever anxiety $(36-50)$.

4-Beck depression inventory ${ }^{[12]}$ : For measuring the existence and the severity of depression. We used the Arabic version translated by AbdelKhalek. ${ }^{[13]}$ It is 21 -question multiplechoice self-report inventory.It has Score range from 0 to 63 :normal $(0-$ 13), mild depression(14 - 19), moderate depression(20 - 28) and sever depression $(29-63)$.

4-Eysenck personality questionnaire (EPQ) ${ }^{[14]}$ :To assess the personality traits of a person .It assess the following personality traits Extraversion (E) and Neuroticism $(\mathrm{N})$, Psychoticism (P) and Lie (L).It Contain 91statements.We used the Arabic version translated by AbdelKhalek $^{[15]}$. 


\section{Ethical consideration:}

Ethical approval was obtained from Scientific Research Ethical Committee of the faculty of medicine, Sohag University. Additionally, official approval from Deans of the fourstudied Faculties was secured. An informed consent was obtained from each participant prior to filling the questionnaires.

\section{The statistical analysis:}

The data were coded, tabulated and analyzed using [SPSS] version 20 .
Qualitative data were expressed as numbers and percentages, and the Chi-square test were applied to test the relationship between variables. Quantitative data were expressed as mean and standard deviation (Mean \pm $\mathrm{SD})$ and t- test were used to compare means for groups. The associations between PIU and the studied variables were examined at both univariate and multivariable levels using logistic regression .A p-value of $<0.05$ were considered as statistically significant.

\section{RESULTS}

The current study included 588 students, 196 student from two practical faculties (faculty of Veterinary Medicine and faculty of Science) and 392 students from Two theoretical faculties (faculty of Arts and faculty of commerce). The mean age of students of practical faculties was 20.19 \pm 1.58 , and for students of theoretical faculties was 20.17 \pm 1.47 with equal number of females and male students (Table 1).Nearly one third of the students were PIU and two thirds were normal internet users. Students of theoretical facultiessignificantly had Potentialy problematic internet use (PIU) thanStudents of practical faculties (Table 2). PIU significantly more in younger age than normal internet user. Male students significantly were PIU than females students. (Table 3). PIU significantly more in theoretical faculties, significantly more in $1^{\text {st }}$ grade also, tended to be high among students of low academic performance. Students with PIUsignificantly had computer and internet at home and used internet in chatting and games (Table 4). 86.96\% of Students with PIU significantly had anxiety (Figure1). $85.02 \%$ of Students with PIUsignificantly had depression (Figure2). Students with PIUsignificantly had high score in Psychoticismand Neuroticismand low score in Lie and Extraversion performance(Table 5).Final logistic regression analysis identified that Depression, Availability of internet and computer at home,Anxiety, high score of Psychoticism, using Internet for chatting, high monthly income , $1^{\text {st }}$ grade , low score of Lie, young age low academic performance, Theoretical faculties andMale genderare strong predictors of PIU.(Table 6). 
SOHAG MEDICAL JOURNAL

Correlation of internet addiction

Vol. 23 No.1 Jan 2019

Osama Abd Alreheem Mahmoud.et al

\begin{tabular}{|c|c|c|c|c|c|}
\hline \multirow{2}{*}{$\begin{array}{l}\text { Socio- } \\
\text { demographic } \\
\text { data }\end{array}$} & \multicolumn{2}{|c|}{ Practical } & \multicolumn{2}{|c|}{ Theoretical } & \multirow{2}{*}{$P$ value } \\
\hline & $\mathrm{N}=196$ & Percentage \% & $\mathrm{N}=392$ & Percentage $\%$ & \\
\hline $\begin{array}{l}\text { Age/years } \\
\text { Mean } \pm \text { SD }\end{array}$ & $20.19 \pm 1.58$ & & $20.17 \pm 1.47$ & & 0.91 \\
\hline $\begin{array}{l}\text { Gender } \\
\text { Females } \\
\text { Males }\end{array}$ & $\begin{array}{l}98 \\
98 \\
\end{array}$ & $\begin{array}{l}50.00 \% \\
50.00 \% \\
\end{array}$ & $\begin{array}{l}196 \\
196 \\
\end{array}$ & $\begin{array}{l}50.00 \% \\
50.00 \% \\
\end{array}$ & 1.00 \\
\hline $\begin{array}{l}\text { Residence } \\
\text { Rural } \\
\text { Urban } \\
\end{array}$ & $\begin{array}{c}70 \\
126 \\
\end{array}$ & $\begin{array}{l}35.71 \% \\
64.29 \% \\
\end{array}$ & $\begin{array}{l}189 \\
203 \\
\end{array}$ & $\begin{array}{l}48.21 \% \\
51.79 \% \\
\end{array}$ & $0.004 * *$ \\
\hline $\begin{array}{l}\text { Residence with } \\
\text { Alone } \\
\text { With parents } \\
\end{array}$ & $\begin{array}{c}42 \\
154 \\
\end{array}$ & $\begin{array}{l}21.43 \% \\
78.57 \% \\
\end{array}$ & $\begin{array}{l}126 \\
266\end{array}$ & $\begin{array}{l}32.14 \% \\
67.86 \% \\
\end{array}$ & $0.007 * *$ \\
\hline $\begin{array}{l}\text { Father job } \\
\text { Farmer } \\
\text { Official employed } \\
\text { Self employed } \\
\text { Retired } \\
\end{array}$ & $\begin{array}{c}19 \\
112 \\
37 \\
28 \\
\end{array}$ & $\begin{array}{c}9.69 \% \\
57.14 \% \\
18.88 \% \\
14.29 \% \\
\end{array}$ & $\begin{array}{c}52 \\
162 \\
105 \\
73 \\
\end{array}$ & $\begin{array}{l}13.27 \% \\
41.33 \% \\
26.79 \% \\
18.62 \% \\
\end{array}$ & $0.004 * *$ \\
\hline $\begin{array}{l}\text { Father education } \\
\text { Illiterate } \\
\text { Primary } \\
\text { Preparatory } \\
\text { Secondary } \\
\text { University }\end{array}$ & $\begin{array}{l}12 \\
19 \\
20 \\
46 \\
99\end{array}$ & $\begin{array}{c}6.12 \% \\
6.69 \% \\
10.20 \% \\
23.47 \% \\
50.51 \% \\
\end{array}$ & $\begin{array}{c}50 \\
29 \\
30 \\
111 \\
172 \\
\end{array}$ & $\begin{array}{c}12.76 \% \\
7.40 \% \\
7.65 \% \\
28.32 \% \\
43.88 \% \\
\end{array}$ & $0.046^{*}$ \\
\hline $\begin{array}{l}\text { Mother education } \\
\text { Illiterate } \\
\text { Primary } \\
\text { Preparatory } \\
\text { Secondary } \\
\text { University }\end{array}$ & $\begin{array}{l}29 \\
16 \\
17 \\
60 \\
74\end{array}$ & $\begin{array}{c}14.80 \% \\
8.16 \% \\
8.67 \% \\
30.61 \% \\
37.76 \% \\
\end{array}$ & $\begin{array}{c}69 \\
44 \\
49 \\
96 \\
134 \\
\end{array}$ & $\begin{array}{l}17.60 \% \\
11.22 \% \\
12.50 \% \\
24.49 \% \\
34.18 \% \\
\end{array}$ & $0.021^{*}$ \\
\hline $\begin{array}{l}\text { Monthly income } \\
\text { Less than } 2000 \\
2000-5000 \\
\text { More than } 5000\end{array}$ & $\begin{array}{l}58 \\
97 \\
41 \\
\end{array}$ & $\begin{array}{l}29.59 \% \\
49.49 \% \\
20.92 \% \\
\end{array}$ & $\begin{array}{l}113 \\
176 \\
103 \\
\end{array}$ & $\begin{array}{l}28.83 \% \\
44.90 \% \\
26.28 \% \\
\end{array}$ & 0.34 \\
\hline
\end{tabular}

Table(1): Socio-demographic data among students of practical and theoretical faculties

\begin{tabular}{|c|c|c|c|c|c|c|c|c|}
\hline \multirow{2}{*}{$\begin{array}{l}\text { internet } \\
\text { addiction } \\
\text { test }\end{array}$} & \multicolumn{2}{|c|}{ Practical } & \multicolumn{2}{|c|}{ Theoretical } & \multicolumn{2}{|c|}{ Total } & \multirow{2}{*}{$\begin{array}{l}X^{2} \\
\text { valu } \\
\text { e }\end{array}$} & \multirow{2}{*}{$\begin{array}{l}P \\
\text { value }\end{array}$} \\
\hline & $\mathrm{N}=196$ & $\begin{array}{l}\text { Percenta } \\
\text { ge } \%\end{array}$ & $\begin{array}{l}\mathrm{N}=39 \\
2\end{array}$ & $\begin{array}{l}\text { Percentage } \\
\%\end{array}$ & $\mathbf{N}$ & $\begin{array}{l}\text { Percent } \\
\text { age } \%\end{array}$ & & \\
\hline \multicolumn{9}{|l|}{$\begin{array}{l}\text { Internet } \\
\text { addiction }\end{array}$} \\
\hline $\begin{array}{l}\text { Non } \\
\text { problematic } \\
\text { (Normal) }\end{array}$ & 143 & $72.96 \%$ & 238 & $60.71 \%$ & 381 & $64.80 \%$ & 8.59 & $\begin{array}{l}0.003 \\
* *\end{array}$ \\
\hline $\begin{array}{l}\text { Potentialy } \\
\text { problematic }\end{array}$ & 53 & $27.04 \%$ & 154 & $39.29 \%$ & 207 & $35.20 \%$ & & \\
\hline
\end{tabular}

Table(2): Distribution of internet addiction among students of practical and theoretical faculties. 
SOHAG MEDICAL JOURNAL

Vol. 23 No.1 Jan 2019
Correlation of internet addiction

Osama Abd Alreheem Mahmoud.et al

\begin{tabular}{|c|c|c|c|c|c|c|}
\hline \multirow{2}{*}{$\begin{array}{l}\text { Socio- } \\
\text { demographic } \\
\text { data }\end{array}$} & \multicolumn{2}{|c|}{ non-problematicuse } & \multicolumn{2}{|c|}{ potentialy problematic use } & \multirow[t]{2}{*}{$\mathrm{X}^{2}$ value } & \multirow[t]{2}{*}{ P value } \\
\hline & $\mathrm{N}=381$ & Percentage \% & $\mathrm{N}=207$ & Percentage \% & & \\
\hline $\begin{array}{l}\text { Age/years } \\
\text { Mean } \pm \text { SD }\end{array}$ & $\begin{array}{c}20.36 \pm \\
1.50\end{array}$ & & $\begin{array}{c}19.85 \pm \\
1.45\end{array}$ & & $\mathrm{t}=4.02$ & $\begin{array}{l}0.0001 \\
* *\end{array}$ \\
\hline $\begin{array}{l}\text { Gender } \\
\text { Females } \\
\text { Males }\end{array}$ & $\begin{array}{l}223 \\
158\end{array}$ & $\begin{array}{l}58.53 \% \\
41.47 \%\end{array}$ & $\begin{array}{c}71 \\
136\end{array}$ & $\begin{array}{l}34.30 \% \\
65.70 \%\end{array}$ & 31.49 & $\begin{array}{l}<0.000 \\
1 * *\end{array}$ \\
\hline $\begin{array}{l}\text { Residence } \\
\text { Rural } \\
\text { Urban } \\
\end{array}$ & $\begin{array}{l}163 \\
218 \\
\end{array}$ & $\begin{array}{l}42.78 \% \\
57.22 \% \\
\end{array}$ & $\begin{array}{c}96 \\
111 \\
\end{array}$ & $\begin{array}{l}46.38 \% \\
53.62 \% \\
\end{array}$ & 0.70 & 0.43 \\
\hline $\begin{array}{l}\text { Residence } \\
\text { with } \\
\text { Alone } \\
\text { With parents } \\
\end{array}$ & $\begin{array}{l}96 \\
285 \\
\end{array}$ & $\begin{array}{l}25.20 \% \\
74.80 \%\end{array}$ & $\begin{array}{l}72 \\
135\end{array}$ & $\begin{array}{l}34.78 \% \\
65.22 \%\end{array}$ & 6.04 & $0.01 *$ \\
\hline $\begin{array}{l}\text { Father job } \\
\text { Farmer } \\
\text { Official } \\
\text { employed } \\
\text { Self } \\
\text { employed } \\
\text { Retired }\end{array}$ & $\begin{array}{c}59 \\
149 \\
106 \\
67\end{array}$ & $\begin{array}{l}15.49 \% \\
39.11 \% \\
27.82 \% \\
17.59 \%\end{array}$ & $\begin{array}{c}12 \\
125 \\
36 \\
34\end{array}$ & $\begin{array}{l}5.80 \% \\
60.39 \% \\
17.39 \% \\
16.43 \%\end{array}$ & 29.61 & $\begin{array}{l}0.0001 \\
* *\end{array}$ \\
\hline 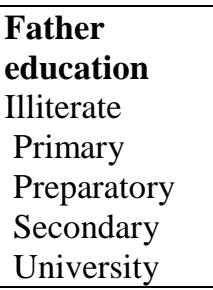 & $\begin{array}{c}54 \\
37 \\
36 \\
100 \\
154\end{array}$ & $\begin{array}{c}14.17 \% \\
9.71 \% \\
9.45 \% \\
26.25 \% \\
40.42 \%\end{array}$ & $\begin{array}{c}8 \\
11 \\
14 \\
57 \\
117\end{array}$ & $\begin{array}{c}3.86 \% \\
5.31 \% \\
6.76 \% \\
27.54 \% \\
56.52 \%\end{array}$ & 25.46 & $\begin{array}{l}<0.000 \\
1 * *\end{array}$ \\
\hline $\begin{array}{l}\text { Mother } \\
\text { education } \\
\text { Illiterate } \\
\text { Primary } \\
\text { Preparatory } \\
\text { Secondary } \\
\text { University } \\
\end{array}$ & $\begin{array}{c}83 \\
42 \\
49 \\
91 \\
116\end{array}$ & $\begin{array}{l}21.78 \% \\
11.02 \% \\
12.86 \% \\
23.88 \% \\
30.45 \%\end{array}$ & $\begin{array}{l}15 \\
18 \\
17 \\
65 \\
92\end{array}$ & $\begin{array}{c}7.25 \% \\
8.70 \% \\
8.21 \% \\
31.40 \% \\
44.44 \%\end{array}$ & 30.59 & $\begin{array}{l}<0.000 \\
1 * *\end{array}$ \\
\hline $\begin{array}{l}\text { Monthly } \\
\text { income } \\
\text { Less than } \\
2000 \\
2000-5000 \\
\text { More than } \\
5000\end{array}$ & $\begin{array}{c}135 \\
188 \\
58\end{array}$ & $\begin{array}{l}35.43 \% \\
49.34 \% \\
15.22 \%\end{array}$ & $\begin{array}{l}36 \\
85 \\
86\end{array}$ & $\begin{array}{l}17.39 \% \\
41.06 \% \\
41.55 \%\end{array}$ & 54.94 & $\begin{array}{l}<0.000 \\
1 * *\end{array}$ \\
\hline
\end{tabular}

Table (3): Comparison between non-problematic and potentialy problematic internet user according to Socio-demographic data. 
SOHAG MEDICAL JOURNAL

Vol. 23 No.1 Jan 2019
Correlation of internet addiction Osama Abd Alreheem Mahmoud.et al

\begin{tabular}{|c|c|c|c|c|c|c|}
\hline \multirow{2}{*}{ Academic data } & \multicolumn{2}{|c|}{ non-problematic use } & \multicolumn{2}{|c|}{$\begin{array}{c}\text { potentialy } \\
\text { problematic use }\end{array}$} & \multirow[t]{2}{*}{$\begin{array}{c}\mathrm{X}^{2} \\
\text { value }\end{array}$} & \multirow[t]{2}{*}{ P value } \\
\hline & $\begin{array}{l}N=38 \\
1\end{array}$ & $\begin{array}{l}\text { Percentage } \\
\%\end{array}$ & $\begin{array}{l}N=20 \\
7\end{array}$ & $\begin{array}{l}\text { Percentage } \\
\%\end{array}$ & & \\
\hline $\begin{array}{l}\text { Faculty } \\
\text { Practical } \\
\text { Therotical }\end{array}$ & $\begin{array}{l}143 \\
238\end{array}$ & $\begin{array}{l}37.53 \% \\
62.47 \%\end{array}$ & $\begin{array}{c}53 \\
154\end{array}$ & $\begin{array}{l}25.60 \% \\
74.40 \%\end{array}$ & 8.59 & $0.003 * *$ \\
\hline $\begin{array}{l}\text { Grade of faculty } \\
1^{\text {st }} \text { grade } \\
4^{\text {th }} \text { grade }\end{array}$ & $\begin{array}{l}167 \\
214\end{array}$ & $\begin{array}{l}43.83 \% \\
56.17 \%\end{array}$ & $\begin{array}{c}126 \\
81\end{array}$ & $\begin{array}{l}60.87 \% \\
39.13 \%\end{array}$ & 15.57 & $<0.0001 * *$ \\
\hline $\begin{array}{l}\text { Academic } \\
\text { performance } \\
\text { Average } \\
\text { Good } \\
\text { Excellent }\end{array}$ & $\begin{array}{c}161 \\
58 \\
162\end{array}$ & $\begin{array}{l}42.26 \% \\
15.22 \% \\
42.52 \%\end{array}$ & $\begin{array}{l}71 \\
73 \\
63\end{array}$ & $\begin{array}{l}34.30 \% \\
35.27 \% \\
30.43 \%\end{array}$ & 31.46 & $<0.0001 * *$ \\
\hline $\begin{array}{l}\text { Presence of } \\
\text { computer at home } \\
\text { Not present } \\
\text { Present } \\
\end{array}$ & $\begin{array}{l}147 \\
234 \\
\end{array}$ & $\begin{array}{l}38.58 \% \\
61.42 \% \\
\end{array}$ & $\begin{array}{c}42 \\
165 \\
\end{array}$ & $\begin{array}{l}20.29 \% \\
79.71 \% \\
\end{array}$ & 20.58 & $<0.0001 * *$ \\
\hline $\begin{array}{l}\text { Availability of } \\
\text { internet at home } \\
\text { Not present } \\
\text { Present }\end{array}$ & $\begin{array}{l}156 \\
225\end{array}$ & $\begin{array}{l}40.94 \% \\
59.06 \%\end{array}$ & $\begin{array}{c}32 \\
175\end{array}$ & $\begin{array}{l}15.46 \% \\
84.54 \%\end{array}$ & 40.06 & $<0.0001 * *$ \\
\hline $\begin{array}{l}\text { Main aim of } \\
\text { internet use } \\
\text { Chatting } \\
\text { Games } \\
\text { Gather information } \\
\text { News }\end{array}$ & $\begin{array}{c}74 \\
61 \\
144 \\
202 \\
\end{array}$ & $\begin{array}{l}19.42 \% \\
16.01 \% \\
37.80 \% \\
26.77 \% \\
\end{array}$ & $\begin{array}{l}87 \\
59 \\
31 \\
30\end{array}$ & $\begin{array}{l}42.03 \% \\
28.50 \% \\
14.98 \% \\
14.49 \% \\
\end{array}$ & 67.77 & $<0.0001 * *$ \\
\hline
\end{tabular}

Table (4): Comparison between non-problematic and potentialy problematic internet user regarding to Academic data and patterns of internet use

\begin{tabular}{|l|c|c|c|l|}
\hline $\begin{array}{l}\text { Eysenck } \\
\text { personality } \\
\text { questionnaire } \\
\text { (EPQ) }\end{array}$ & $\begin{array}{c}\text { Non- } \\
\text { problematic } \\
\text { use }\end{array}$ & $\begin{array}{c}\text { Potentialy } \\
\text { problematic } \\
\text { use }\end{array}$ & Z value & P value \\
\cline { 2 - 5 } & $\mathbf{N = 3 8 1}$ & $\mathbf{N = 2 0 7}$ & \\
\hline $\begin{array}{l}\text { Psychoticism } \\
\text { Mean } \pm \text { SD }\end{array}$ & $5.97 \pm 3.57$ & $13.18 \pm 6.40$ & -12.75 & $<0.0001^{* *}$ \\
\hline $\begin{array}{l}\text { Extraversion } \\
\text { Mean } \pm \text { SD }\end{array}$ & $12.39 \pm 3.98$ & $8.27 \pm 4.66$ & 0.90 & $<0.0001^{* *}$ \\
\hline $\begin{array}{l}\text { Neuroticism } \\
\text { Mean } \pm \text { SD }\end{array}$ & $11.08 \pm 5.63$ & $16.53 \pm 3.67$ & -11.12 & $<0.0001^{* *}$ \\
\hline $\begin{array}{l}\text { Lie } \\
\text { Mean } \pm \text { SD }\end{array}$ & $15.33 \pm 3.98$ & $9.13 \pm 4.65$ & 13.69 & $<0.0001^{* *}$ \\
\hline
\end{tabular}

Table(5):Comparison between non-problematic and potentialy problematic internet user regarding to the personality traits 


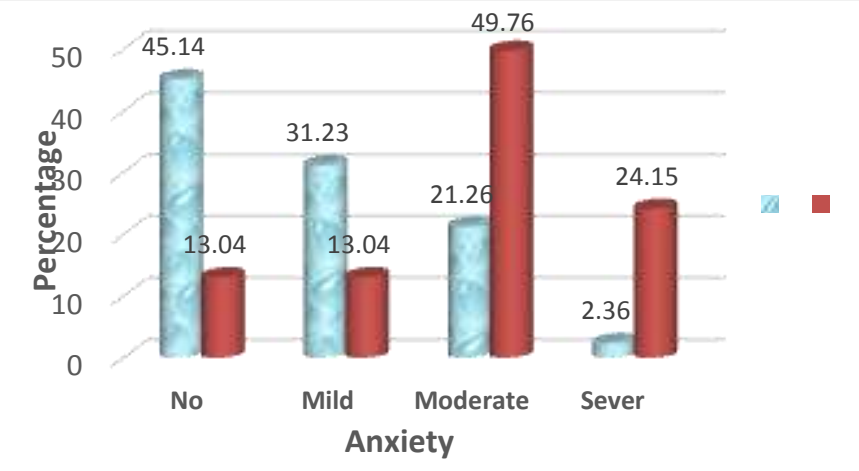

Figure (1): Comparison between non-problematic and potentialy problematic internet user regarding to anxiety.

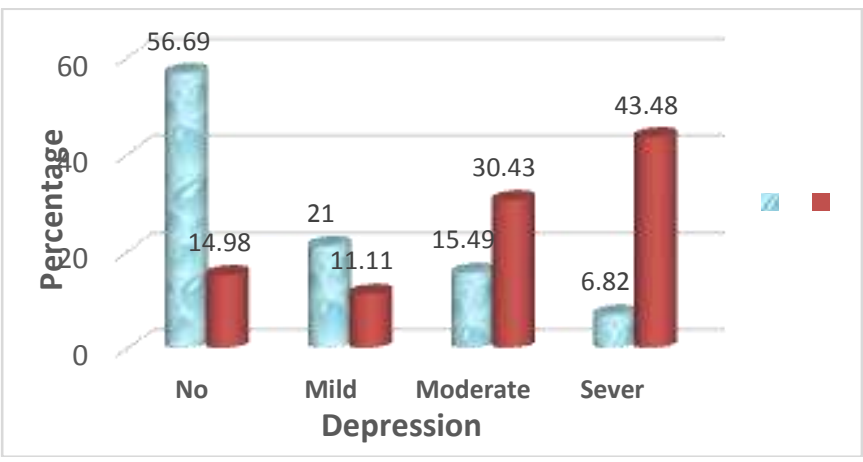

Figure (2): Comparison between non-problematic and potentialy problematic internet user according to depression. 
SOHAG MEDICAL JOURNAL

Vol. 23 No.1 Jan 2019
Correlation of internet addiction

Osama Abd Alreheem Mahmoud.et al

\begin{tabular}{|c|c|c|}
\hline Variable & $\begin{array}{c}\text { Odds ratio }(95 \% \\
\text { confidence } \\
\text { interval })\end{array}$ & $P$ value \\
\hline Sever vs. no Depression & $6.43(2.40-17.25)$ & $<0.0001 * *$ \\
\hline Availability of internet at home & $5.25(2.55-10.81)$ & $<0.0001 * *$ \\
\hline Moderate vs. no Anxiety & $4.85(2.00-11.75)$ & $<0.0001 * *$ \\
\hline Moderate vs. no Depression & $4.49(1.93-10.44)$ & $<0.0001 * *$ \\
\hline Presence of computer at home & $2.21(1.43-3.42)$ & $<0.0001 * *$ \\
\hline Psychoticism & $1.21(1.11-1.32)$ & $<0.0001 * *$ \\
\hline Gather information vs. Chatting & $0.23(0.11-0.47)$ & $<0.0001 * *$ \\
\hline $\begin{array}{l}\text { Monthly income } \\
\text { More than } 5000 \text { vs. Less than } \\
2000\end{array}$ & $3.82(2.22-6.58)$ & $<0.001 * *$ \\
\hline $4^{\text {th }}$ grade vs. $1^{\text {st }}$ grade & $0.44(0.30-0.66)$ & $<0.001 * *$ \\
\hline Lie & $0.87(0.79-0.94)$ & $0.001 * *$ \\
\hline Age/years & $0.73(0.59-0.88)$ & $0.002 * *$ \\
\hline Sever vs. noAnxiety & $8.01(2.06-31.10)$ & $0.003 * *$ \\
\hline $\begin{array}{l}\text { Academic performance } \\
\text { Excellent vs. good }\end{array}$ & $0.50(0.30-0.83)$ & $0.007 * *$ \\
\hline Theoretical vs. Practical & $2.17(1.18-4.01)$ & $0.01 *$ \\
\hline $\begin{array}{l}\text { Academic performance } \\
\text { Average vs. good }\end{array}$ & $0.53(0.32-0.87)$ & $0.01 *$ \\
\hline $\begin{array}{l}\text { Aim of internet use } \\
\text { News vs. Chatting }\end{array}$ & $0.39(0.18-0.82)$ & $0.01 *$ \\
\hline Males Vs. females & $2.18(1.14-4.18)$ & $0.02 *$ \\
\hline $\begin{array}{l}\text { Monthly income } \\
2000-5000 \text { vs. Less than } 2000\end{array}$ & $1.65(1.03-2.65$ & $0.04 *$ \\
\hline
\end{tabular}

Table (6): Final logistic regression model of factor affecting presence of potential problematic internet use.

\section{DISCUSSION}

Our study was conducted to assess the prevalence of PIU among undergraduate university students and to determine the personal and social characteristics of PIU among adolescents. We found that prevalence rate ofPIU among students was $35.20 \%$. Our findings were consistent with the results of studies conducted in Greek $34.7 \%^{[16]}$ and in Korea 38\% $\cdot{ }^{[17]}$ Another study done in Sohag university, revealed that $47.7 \%$ of the surveyed medical students suffered from IA. ${ }^{[18]}$ Also the results of our study were slightly lower than fromthe results of ${ }^{[19]}$ and ${ }^{[20]}$ in India, they reported that $46.8 \%$ and $44.6 \%$ of the respondent had Internet addiction respectively. 
SOHAG MEDICAL JOURNAL

Vol. 23 No.1 Jan 2019

In the present study, we found that Students of theoretical facultiessignificantly had PIU (39.29\%)thanStudents of practical faculties $(27.04 \%)$. These findings are consistent with the results of many studies done in Egypt and found that the Literary colleges, were more likely to suffer more from PIU than scientific colleges . ${ }^{[21,22,23]}$ This marked difference could be explained by the academic overload and the high pressure of study demands in students of practical faculties. ${ }^{[24]}$

Regarding age, the PIU significantly more in younger age than normal internet user. Most of the previous researchers had concluded that younger people are at higher risk for internet addiction disorder.$^{[6,25,26]}$ This was not in agreement with lee and Stapinski ${ }^{[27]}$ who could not report a significant association between age and internet addiction.

PIU was significantly more in males than in femalesand this was in line with those of most of the previous studies, found that male gender is a predictor of IA ${ }^{[6,28,29]}$ Similar findings were reported by studies conducted among students in Iran ${ }^{[30]}$, China ${ }^{[31]}$ and Japan ${ }^{[32]}$. Studies have shown that the link between male gender and internet addiction may be mediated by the online activities that they were engaged upon. ${ }^{[33]}$ Activities such as online gaming which are highly addictive were mainly reported among males, whereas females were more involved in social networking. ${ }^{[34]}$

Regarding father job, Fathers ofPIU users significantly employed. Also, PIU significantly higher in students with high monthly income.Internet addiction was higher among students whose mothers and fathers were employed and among those with a high family income level.$^{[23,35,36]}$

Our study showed that incidence of PIU tended to be higher among
Correlation of internet addiction

Osama Abd Alreheem Mahmoud.et al

students of low academic performance. The results of the present study were in agreement with [23,37] who found significant negative correlation was present between IAT scores and academic grades of the students. Students with PIU,significantly had computer and internet at home thanStudents of normal internet use. The same findings are reported with the results of many studies done in Egypt. ${ }^{[37,38]}$ Regarding main aim if internet use PIU, significantly used internet in chatting and games. In many studies, it was found that addicted people spend more time on websites with gaming, and chatting content $\cdot{ }^{[39,40,41]}$ Online activities and practices have also, been found to be important factors in detecting internet addiction $\cdot{ }^{[42]}$ The results of our study also Similar to the findings of .${ }^{[29,43]}$ who revealed that undergraduate problematic Internet users were more likely to use real-time interactive activities such as chat and online games.

We found that $86.9 \%$ of Students with PIUsignificantly had anxiety. Previous reports demonstrated that anxiety, ADHD, and depression are the 3 psychological disorders that are prevalent in individuals with internet addiction ${ }^{[44]}$ Also, other researchers have found that shyness and social anxiety are significant predictors of internet addiction . ${ }^{[4,46,47]}$ Regarding depression85.02\% of Students with PIU significantly had Depression. Previous studies showed a significant association between internet addiction (IA) and depression among

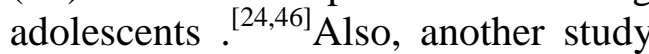
reported a significant association between the various level of internet addiction and negative emotions, such as depression, loneliness, and obsessive behavior . ${ }^{[17,48]}$ 
The study showed that Students with PIU significantly had high score in Psychoticism and Neuroticism and low score in Lie and Extraversion performance. This was in agreement with $\mathrm{Ge}$ et al. ${ }^{[49]}$ who revealed that there was a positive correlation between the total scores of internetaddiction and psychoticism.Also,our results were in line with Dong et al. ${ }^{[50]}$ whofound that internet addicts showed significantly higher $\mathrm{N}$ score and lower Lie scores than those in the control group. Zamaniet al. ${ }^{[51]}$ found negative and significant relationship observed between Internet addiction and extroversion.

\section{LIMITATIONS:}

One of the limitations of this study is that data collection was based on selfreported questionnaires so prone to recall bias. Another limitation is being a cross-sectional study which showed the relation between PIU and some potential risk factors without being able to conclude a cause-effect relationship.

\section{CONCLUSION RECOMMENDATIONS:}

AND

This is one of the studies that assess the prevalence of PIU among undergraduate university studentsin Egypt.Nearly one third of the students were PIU andPIU significantly more in theoretical faculities than in practical faculities. The study revealed that young age, male gender, $1^{\text {st }}$ grade,low academic performance, high and moderate monthly income, presence of computer at home, Availability of internet at home, using Internet for chatting and gaming,Anxiety, depression and high score in Psychoticism and low score in Lie arestrong predictors of PIU. We recommend Counseling to help the university students to learn social skills and healthier ways of coping with uncomfortable emotions, such as stress, anxiety, or depression.

\section{REFERENCES}

1.Young KS. Caught in the Net: How to Recognize the Signs of Internet Addiction and a Winning Strategy for Recovery. New York, NY: J. Wiley.1998.

2. Young KS. Internet addiction. Am Behav Sci. 2004;48: 402-415.

3.Weinstein, A., Lejoyeux, M. Internet addiction or excessive internet use. Am J Drug Alcohol Abuse.2010;36:277-283.

4.Chakraborty, K., Basu, D., Vijaya et al.Internet addiction: consensus, controversies, and the way ahead. East Asian Arch Psychiatry.2010; 20(3):123-32.

5.Lam LT, Peng ZW, Mai JC, Jing J. Factors associated with Internet addiction among adolescents. CyberpsycholBehav .2009; 12(5): 551-5.

6.Tsai, H.F., Cheng, S.H., Yeh, T.L, et al.The risk factors of Internet Addiction.a survey of university freshmen. Psychiatry Research.2009;167:294-299.

7.Cao, F., \& Su, L.Internet addiction among Chinese adolescents: prevalence and psychological features. Child: Care, Health\& Development.2007;33:275-281.

8.Mehroof, M., \& Griffiths, M.D.Online gaming addiction: the role of sensation seeking, self-control, neuroticism, aggression, state anxiety, and trait anxiety. Cyber Psychology, Behavior, \& Social Networking.2010; 13:313-316.

9.Hawi ,N. S. Edcyber psychology, behavior, and social networking Mary Ann Liebert.2013; 16, 3.

10.Taylor, J."A personality scale of manifest anxiety". The Journal of Abnormal and Social Psychology.1953; 48(2): 285-290.

11.Fahmi, M., Ghali, M., \&Meleka, K. Arabic version of the personality scale of manifest anxiety, Egyptian Psychiatry.1977; 11: 119- 126.

12.Beck, A.T, \&Steer, R.A. Manual for the Beck Depression Inventory.San Antonio, TX: Psychological Corporation.1993. 
13.Abdel-Khalek, A.M. Internal consistency of an Arabic adaptation of the Beck Depression Inventory in four Arab countries. Psychol Rep.1988; 82(1):264-266.

14.Eysenck, H. J., \& EysenckSybil, B. G. Manual of the Eysenck Personality Questionnaire. London: Hodder and Stoughton.1975.

15.Abdel-Khalek, A. M. . The Arabic manual of the Eysenck Personality Questionnaire (Children and Adults).Alexandria :Dar Al-Maaref Al-Jamiiyah, in Arabic.1991.

16.Christos, C.F., Constantinos, C.F.,\& Ioannis, S. Problematic Internet Use Among Greek University Students: An Ordinal Logistic Regression with Risk Factors of Negative Psychological Beliefs, Pornographic Sites, and Online Games. Cyberpsychology, Behavior, and Social Networking.2011; 14(1-2):518.

17.Kim, K., Ryu, E., Chon, M-Y, et al. Internet addiction in Korean adolescents and suicidal ideation: A questionnaire survey. International Journal of Nursing Studies.2006; 43(2): 185-192.

18.Ali,Y.H.,Mohammed,A.N.,\&Ali,A.R. Internet addiction among medical students of Sohag University, Egyp. ISSN.2017;2(92) :0013-2446.

19.Nath, K., Naskar, S., \&Victor, R. A. Cross-Sectional Study on the Prevalence, Risk Factors, and Ill Effects of Internet Addiction Among Medical Students in Northeastern India. Prim Care Companion CNS Disord.2016;18(2).

20.Pramanik, T., Sherpa, M.T.,\& Shrestha, R. Internet addiction in a group of medical students: a cross sectional study. Nepal Med Coll J.2012;14(1):46-8.

21.Zeinab ,E.H., Heba, M. A .,Hanaa ,A.E, et al . Problematic Internet Use Among Al-Azhar University Students in Cairo.The Egyptian Journal of Hospital Medicine .2015; 61: 535547.

22.Desouky, D.A,. \&Ibrahem, R.A. Internet addiction and psychological morbidity among menoufia university students, Egypt. American Journal of Public Health

Research.2015;3(5):192-8.

23.ELNahas,G., AboElella,E., Hewedi,D, et al. Problematic Online Gaming Among a Sample of University Students in Egypt .Addict Disord Their Treatment. www.addictiondisorders.com.2018; 00:000-000.

24.Alavi ,S.S., Maracy, M.R., Jannatifard, F., \&Eslami, M. The effect of psychiatric symptoms on the internet addiction disorder in Isfahan's University students. J Res Med Sci .2011;16:793-800.

25.Dargahi, H., \&Razavi, M. Internet addiction and its related factors in inhabitants, Tehran. Payesh.2007;6(3):265-72.

26. Ghamari, F., Mohammad, B. A., Mohammad ,S. N.,\&Hashiani, A.A. Internet addiction and modeling its risk factors in medical students, iran. Indian J Psychol Med.2011;33(2):15862.

27.Lee, B.W., \&Stapinski,L.A. Seeking safety on the internet: Relationship between social anxiety and problematic internet use. J Anxiety Disord.2012;26(1):197-205.

28.Byun, S., Ruffini, C., Mills, J.E, et al. Internet addiction: metasynthesis of 1996-2006 quantitative research. CyberpsycholBehav .2009;12:203-7.

29. Frangos, C., Frangos, C., \&Kiohos, A. Internet addiction among greek university students: demographic associations with the phenomenon, using the greek version of young's internet addictio. Int $\mathbf{J}$ Econ SciAppl Res .2010;1:49-74.

30.Mazhari,S.The Prevalence of Problematic Internet Use and the Related Factors in Medical Students, Kerman, Iran .Addict Health.2012; 4 (3-4): 87-94.

31.Wang, L., Luo, J., Bai, Y, et al. Internet addiction of adolescents in China: Prevalence, predictors, and association with well-being. Addict Res Theory.2013; 21: 62-9.

32.Sato, $\mathrm{T}$. Internet Addiction among Students: Prevalence and Psychological Problems in Japan. 
SOHAG MEDICAL JOURNAL

Vol. 23 No.1 Jan 2019

Japan Medical Association Journal .2006;49:7-8

33.Kuss, J.D., Griffiths, D.M., Karila, L et al.Internet addiction: a systematic review of epidemiological research for the last decade. Current Pharmaceutical Design.2014;20:40264052.

34.Heo, J., Oh, J., Subramanian, S.V, et al. Addictive internet use among Korean adolescents: a national survey. PloS One .2014;9(2):e87819.

35.Ozgul ,O., Ozlem, O., Alaettin, U. S., \&Sinan, O. Evaluation of Internet Addiction and Depression Among University Students Procedia - Social and Behavioral Sciences.2013;82 : $445-454$.

36.Jackson, L., Alexander, E., Biocca, F, et al. Personality, cognitive style, demographic characteristics and internet use. Findings from the HomeNetToo project. Swiss Journal of Psychology.2003; 62: 79-90.

37.Saied,S.M.,Elsabagh,H.M.,\&ElAfandy,A.M.Internet and facebook addiction among Egyptian and Malaysian medical students .Int $\mathbf{J}$ Community Med Public Health.2016;3(5):1288-1297.

38.Reda, M., Rabie, M., Mohsen, N., \& Hassan, A. Problematic internet users and psychiatric morbidity in a sample of Egyptian adolescents. Psychology.2012;3(8):626-31.

39.Günüç, S., \&Kayri, M. The profile of internet dependency in Turkey and development of internet addiction scale: study of validity and reliability. Hacettepe University Journal of Education .2010;39: 220-232.

40.Kheirkhah, F., Juibary, A.G., \&Gouran, A. Internet addiction, prevalence and epidemiological features in Mazandaran province, Northern Iran. Iran Red Crescent Med J .2010; 12: 133-137.

41.Tahiroğlu, A.Y., Celik, G.G., Fettahoğlu, C, et al. Problematic internet use in the psychiatric sample compared community sample. Turk Neuropsychiatric Society.2010; 47: 241-246.

42.Canbaz, S., Sunter, A.T., Peksen, Y., Canbaz, M. Prevalence of pathological
Correlation of internet addiction

Osama Abd Alreheem Mahmoud.et al

internet use in a sample of Turkish school adolescents. Iran $\mathbf{J}$ Public Health.2009;38:64-71.

43.Caplan, S., Williams, D., \& Yee, N. Problematic Internet use and psychosocial wellbeing among MMO players. Computers in Human Behavior.2009;25:1335-1342.

44.Ko, C. H., Yen, J. Y., Chen, C. C, et al.Gender differences and related factors affecting online gaming addiction among Taiwanese adolescents. Journal of Nervous \& Mental Disease.2005; 193: 273-277.

45.Yen, J., Ko, C., \& Yen, C. The comorbid psychiatric symptoms of internet addiction: attention deficit and hyperactivity disorder (ADHD), depression, social phobia, and hostility. J Adolesc Health.2007; 41, 93-8.

46.Ko, C. H., Yen, J. Y., Chen, C. S, et al. Predictive Values of Psychiatric Symptoms for Internet Addiction in Adolescents. Archives of Pediatrics and

Adolescent Medicine.2009;163(10): 937-943.

47.Yen, C.F., King, B.H., \&Tang, T.C. The association between short and long nocturnal sleep durations and risky behaviours and the moderating factors in Taiwanese adolescents. Psychiatry Res .2010; 179: 69-74.

48.Ha, J. H., Kim, S. Y., Bae, S. C, et al. Depression and Internet addiction in adolescents.

Psychopathology.2007;40(6):424430.

49.Ge,Y., Se,J., \& Zhang, J. Research on Relationship Among InternetAddiction, PersonalityTraits and Mental Health of Urban Left-Behind Children. Global Journal of Health Science.2015;7(4).

50.Dong,G., Wang,J., Yang,X., \&Zhou,H. Risk personality traits of Internet addiction. Asia-Pacific Psychiatry.2013; 316-321.

51.Zamani,B., Abedini,Y.,Kheradmand,A .Internet Dependency and Personality Traits Addict \& Health, Summer \& Autumn .2011; 3: 3-4. 